\title{
Inquadramento diagnostico e clinico dell'asimmetria funzionale
}

\section{Diagnostic and clinical evaluation of functional asymmetry}

Ricevuto il

28 luglio 2013

Accettato il

14 gennaio 2014

*Autore di riferimento

Claudia Santariello

claudiasantariello@msn.com

\section{RIASSUNTO}

OBIETTIVI. Scopo del lavoro è fornire gli elementi per diagnosticare correttamente un'asimmetria mandibolare di tipo funzionale.

MATERIALI E METODI. Attraverso una revisione della letteratura viene analizzata l'asimmetria funzionale con particolare riferimento alle caratteristiche cliniche e muscolari e alle cause determinanti la malocclusione.

RISULTATI E CONCLUSIONI. L'asimmetria funzionale è un quadro clinico che si manifesta in soggetti in crescita. L'esame obiettivo, l'analisi radiografica e lo studio dei modelli in gesso permettono di effettuare una diagnosi adeguata al fine di ricondizionare la crescita del paziente in una direzione armonica.

\section{PAROLE CHIAVE}

- Asimmetria mandibolare

- Asimmetria funzionale

- Cross-bite

- Malocclusione

- Ortodonzia
ABSTRACT

OBJECTIVES. The aim of this paper is to provide the tools to accurately diagnose a functional mandibular asymmetry.

MATERIALS AND METHODS. Several studies in the literature were reviewed, evaluating clinical and muscular features of functional asymmetry and its etiology.

RESULTS AND CONCLUSIONS. Dental interferences in children represent the main etiological factor for functional asymmetry. Clinical examination, $X$-ray analysis and dental casts are essential for an adequate diagnosis and treatment planning to influence the developmental process toward to a more harmonious growth.

KEY WORDS

- Mandibular asymmetry

- Functional asymmetry

- Cross-bite

- Malocclusion

- Orthodontics 


\section{INTRODUZIONE}

Le asimmetrie mandibolari funzionali che si verificano durante la crescita sono il risultato di un'interferenza occlusale che forza la mandibola a deviare lateralmente per raggiungere in massima intercuspidazione una buona stabilità attraverso il maggior numero di contatti occlusali [1-5].

La persistenza della deviazione mandibolare funzionale provoca uno sviluppo disarmonico delle ossa del cranio e, con il tempo, la determinazione di dissimmetrie scheletriche. Una delle strutture maggiormente interessate da tale adattamento è il condilo, con ripercussioni sulla funzionalità dell'articolazione temporo-mandibolare e sulla crescita mandibolare [6].

È quindi fondamentale saper riconoscere e risolvere precocemente un'asimmetria funzionale. Scopo di questo articolo è analizzare tale condizione da un punto di vista clinico-diagnostico al fine di fornire al lettore tutti gli elementi per effettuare una diagnosi e una terapia adeguate, dirette sia all'allontanamento delle cause determinanti la patologia, sia al controllo della sua evoluzione.

\section{DEFINIZIONE E ASPETTI CLINICl}

L'asimmetria funzionale è una condizione clinica caratterizzata dallo scivolamento obbligato della mandibola in direzione laterale durante i movimenti di chiusura [7]. Se tale condizione si mantiene nel tempo determina una serie di alterazioni strutturali a livello istologico, anatomico, muscolare e clinico [4,6,8-15].

Come dimostrano gli studi di Fuentes et al. [9], confermati da Sato et al. [8], in età evolutiva il dislocamento latera- le della mandibola può condurre a un cambiamento adattativo del pattern di crescita del condilo e a una modificazione della sua forma. In particolare Sato et al. [8] prendono in esame le sezioni istologiche della cartilagine condilare di 50 ratti dopo aver applicato agli animali un dispositivo che forza la mandibola a deviare verso un lato. Lo studio dimostra che la deviazione funzionale della mandibola è associata a una crescita differenziale a livello della cartilagine condilare.

Negli animali sottoposti a laterodeviazione forzata per un mese, dopo 2 settimane dall'applicazione del dispositivo lo spessore della cartilagine del condilo presenta una decisiva riduzione nel lato della deviazione, e un graduale aumento nel lato opposto, rispetto al gruppo di controllo. Si assiste quindi a una crescita mandibolare asimmetrica caratterizzata da una minore lunghezza del ramo omolaterale alla deviazione e una maggiore lunghezza del ramo opposto. Tuttavia negli animali in cui l'apparecchio viene sospeso dopo 2 settimane si ottiene il recupero della condizione iniziale a livello delle cartilagini condilari che, alla quarta settimana, presentano lo stesso spessore del gruppo di controllo. Gli autori sottolineano, dunque, l'importanza di eliminare precocemente lo shift mandibolare al fine di riequilibrare la crescita $[8,9]$.

L'asimmetria funzionale è inoltre sostenuta dal sistema neuromuscolare. Come riportato in alcuni studi elettromiografici, l'attività dei muscoli masticatori (massetere, temporale anteriore e posteriore) di pazienti con cross-bite posteriore monolaterale e laterodeviazione funzionale della mandibola differisce rispetto a quella di pazienti con un'occlusione corretta. Esiste, infatti, un'interessante as- sociazione tra il cross-bite monolaterale e un'attività muscolare asimmetrica, che si riscontra sia quando la mandibola è in posizione di riposo sia durante la masticazione e la deglutizione [11-15].

In posizione di riposo il muscolo temporale anteriore e il massetere del lato del cross-bite presentano un'attività elettromiografica maggiore rispetto al lato controlaterale e al gruppo di controllo [11-15]. Il muscolo temporale posteriore assume non solo un'attività asimmetrica sui due lati, ma risulta essere il muscolo con l'attività elettromiografica più intensa rispetto agli altri muscoli analizzati, confermando l'importante ruolo di posizionatore e stabilizzatore della mandibola a riposo $[11,14,15]$.

Durante la masticazione i pazienti con laterodeviazione della mandibola mostrano una predominante attività del muscolo temporale anteriore rispetto al massetere, che permette alla mandibola di raggiungere la stabilità occlusale più idonea per generare la forza masticatoria necessaria, evitando le interferenze occlusali. Il riflesso inibitorio che protegge il sistema stomatognatico dai traumi occlusali conseguenti alle interferenze dentali determina, infatti, una minore attività del massetere rispetto al gruppo di controllo, producendo una forza masticatoria minore [14-16].

Durante la deglutizione i pazienti con cross-bite presentano una minore attività dei muscoli temporali anteriori e dei masseteri a favore di una maggiore attività dei muscoli sopraioidei, caratteristica di uno schema di deglutizione atipico [15].

Le asimmetrie funzionali, infine, si manifestano clinicamente sul piano sagittale e trasversale, mentre sono assenti le caratteristiche tipiche di un'asimmetria sul piano verticale. All'esame obiettivo 
$1 \mathrm{C}$

$1 a$

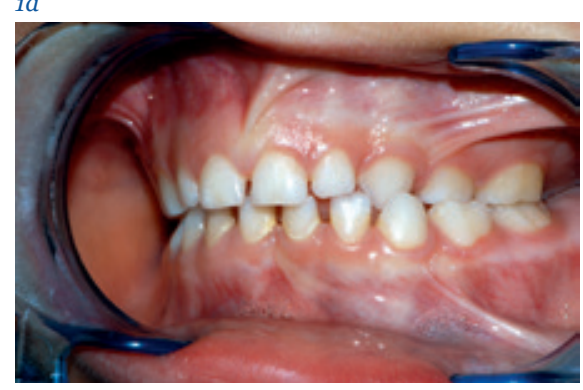

$1 \mathrm{~A}$

$1 b$

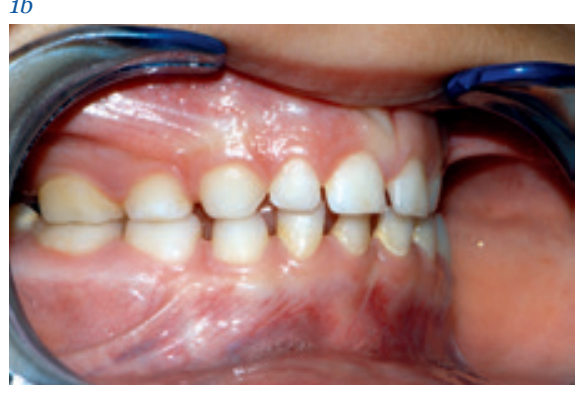

1B

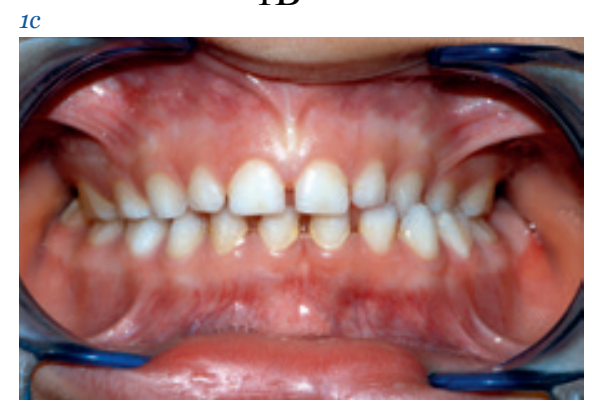

Fig. 1a-c Occlusione di un paziente con asimmetria funzionale. In visione frontale (a) si nota la mancata coincidenza delle linee mediane superiore e inferiore con cross-bite monolaterale a sinistra. In visione laterale si rileva la presenza di un rapporto occlusale sagittale asimmetrico con una Classe II maggiore sul lato del cross-bite (b) rispetto al controlaterale (c)

extraorale si riscontrano una deviazione del mento e un angolo goniaco omolaterale alla laterodeviazione posizionato posteriormente rispetto al controlaterale. A livello intraorale è generalmente presente un cross-bite monolaterale associato a una deviazione della linea interincisiva inferiore verso il lato del cross-bite e a rapporti occlusali sagittali asimmetrici, con una Classe II piena o parziale sul lato del cross-bite e una Classe I sul lato opposto. L'arcata mascellare risulta invece simmetrica rispetto alla base apicale di appartenenza (fig. 1a-c) [6,10].

\subsection{EZIOLOGIA}

La causa dell'insorgenza dell'asimmetria funzionale è esclusivamente di tipo acquisito; il principale fattore eziologico è rappresentato dai precontatti dentali, responsabili delle laterodeviazioni funzionali di lieve entità. Tali interferenze occlusali sono dovute all'inclinazione errata, alla rotazione di un elemento dentale o alla permanenza dell'elemento deciduo in arcata durante l'eruzione del permanente corrispondente. Nella maggior parte dei casi il canino deciduo risulta essere l'elemento dentale più coinvolto [17].

La manifestazione clinica di un'asimmetria funzionale conseguente a precontatti dentali è il cross-bite monolaterale posteriore, espressione di una malocclusione complessa caratterizzata da un deficit simmetrico del mascellare che determina una discrepanza dei rapporti occlusali sul piano trasversale, coinvolgendo uno o più elementi dentali. Tale malocclusione è spesso associata alla presenza di abitudini viziate, quali la deglutizione atipica, la suzione del ciuccio o del dito o la respirazione orale, che modificano l'equilibrio tra le forze esercitate dalla muscolatura esterna delle labbra e delle guance e quelle sviluppate dalla muscolatura interna della lingua, che non svolge la sua azione plasmante sul mascellare superiore [6,10,18-20].

Il cross-bite si ripercuote anche sulla funzione, causando un'inversione dei cicli masticatori. In condizioni normali, sul piano frontale il pattern fisiologico del ciclo masticatorio è caratterizzato da uno spostamento della mandibola verso il basso durante la fase di apertura e da una deviazione dalla parte del bolo nella fase di chiusura. Per esempio, durante la masticazione a destra i cicli masticatori hanno prevalentemente direzione di chiusura oraria, mentre se la masticazione è a sinistra i cicli masticatori hanno prevalentemente direzione di chiusura antioraria. In presenza di un cross-bite monolaterale posteriore, invece, la mandibola compie un ciclo con direzione di chiusura inversa, al fine di non danneggiare le varie componenti dell'apparato stomatognatico coinvolte nella masticazione e di utilizzare una superfice dentale maggiore, con conseguente aumento della capacità masticatoria [21-23].

\subsection{DIAGNOSI}

L'asimmetria funzionale è una malocclusione che coinvolge esclusivamente il paziente in crescita e se diagnosticata correttamente può essere risolta con successo.

Per individuare tale alterazione occlusale è indispensabile uno studio accurato del paziente attraverso l'esame clinico, la valutazione radiografica e l'analisi dei modelli in gesso.

\section{> Esame clinico extraorale}

L'osservazione del volto permette di verificare la presenza di armonia e simmetria tra le componenti facciali. Come descritto da Proffit [17] un viso proporzionato può essere diviso in cinque parti uguali: un quinto centrale delimitato da due rette passanti per l'angolo interno degli occhi, due quinti mediali definiti dalle rette passanti per l'angolo interno ed esterno degli occhi e due quinti laterali 
esterni a questi ultimi. Il naso e il mento devono coincidere con il quinto centrale e l'ampiezza del naso può essere uguale o leggermente più ampia rispetto al quinto stesso. La distanza interpupillare, infine, deve coincidere con l'ampiezza della bocca [17] (fig. 2).

Secondo Arnett et al. [24] la simmetria del volto può essere verificata considerando la posizione del mento rispetto a un piano verticale mediano, definito da una retta passante per la glabella e il filtro. In condizioni di simmetria il mento giace lungo la linea mediana così individuata (fig. 3).

La mancata coincidenza del mento con il quinto centrale del volto o del pogonion cutaneo con la linea mediana del viso individuano una deviazione laterale del mento e sono riscontrabili nel paziente

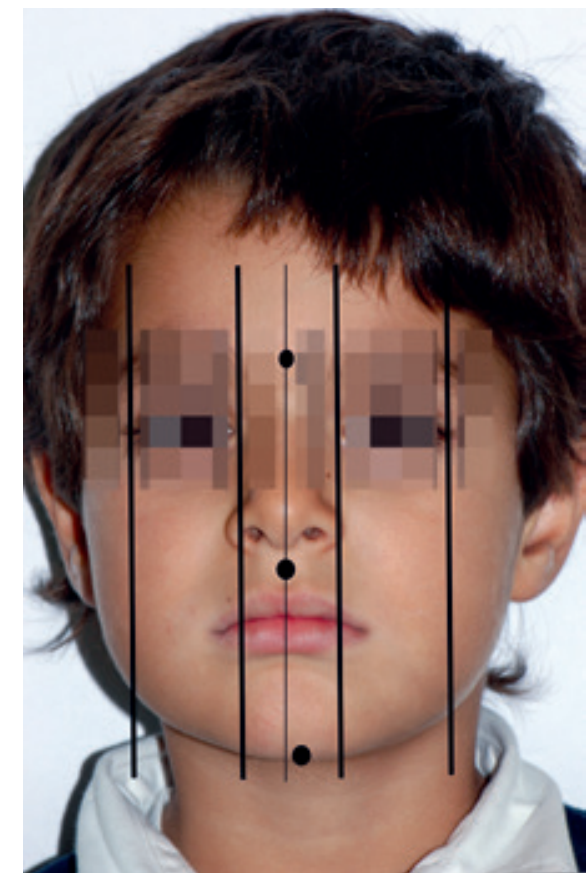

Fig. 2 Visione frontale di un paziente con asimmetria funzionale: mancata coincidenza del mento con il quinto centrale del volto con un'asimmetria del terzo inferiore del viso di tipo funzionale.

\section{$>$ Esame clinico intraorale}

Una volta verificata la presenza di una deviazione mandibolare si osserva il paziente in occlusione per esaminare la posizione delle linee mediane superiore e inferiore.

In massima intercuspidazione si valuta:

- la linea mediana superiore rispetto a quella inferiore, e viceversa;

- la posizione delle singole mediane delle due arcate rispetto alla mediana del viso;

- la posizione delle singole mediane rispetto alla linea mediana della base apicale di appartenenza.

In presenza di una deviazione funzionale della mandibola generalmente si riscon-

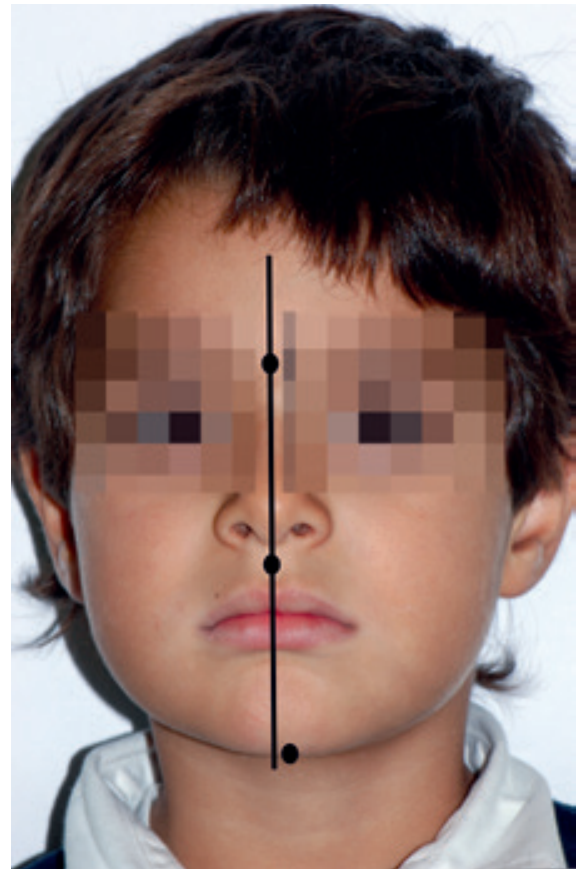

Fig. 3 Visione frontale di un paziente con asimmetria funzionale: mancata coincidenza del mento con la linea verticale mediana del volto tra una mancata coincidenza reciproca delle linee mediane, con una deviazione della linea mediana inferiore rispetto al centro del viso (fig. 4).

La valutazione della coincidenza delle mediane in apertura permette di fare diagnosi differenziale tra un'asimmetria funzionale e un'asimmetria vera (scheletrica o dentale). In particolare:

- nell'asimmetria vera l'assenza di coincidenza delle linee mediane persiste sia in massima intercuspidazione sia in apertura;

- nella deviazione mandibolare funzionale dovuta a un'interferenza occlusale, le linee mediane non sono coincidenti in chiusura ma lo diventano in apertura [17,25,26] (fig. 5a-c).

Guidando la mandibola in relazione centrica è possibile individuare con certezza

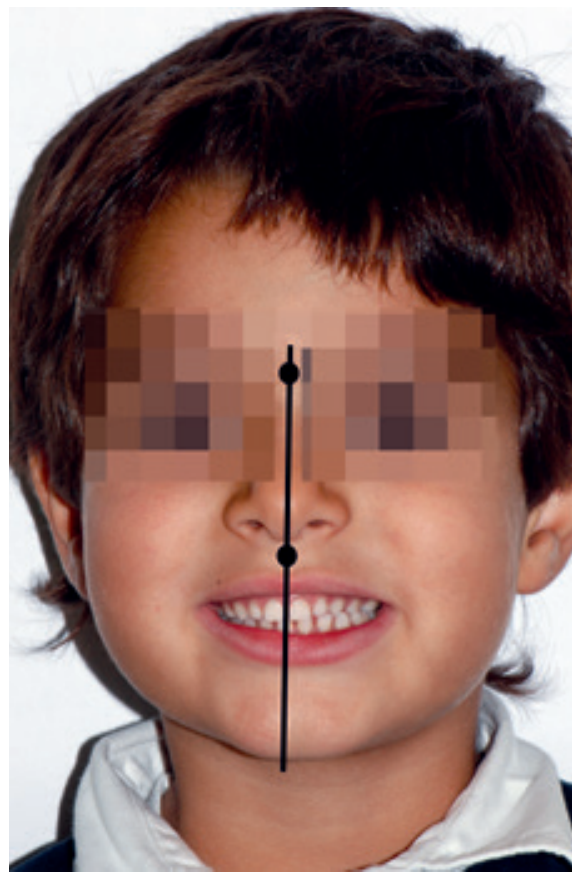

Fig. 4 In presenza di un'asimmetria funzionale la mancata coincidenza delle linee mediane superiore e inferiore è riconducibile a una deviazione della linea mediana inferiore rispetto al centro del volto 

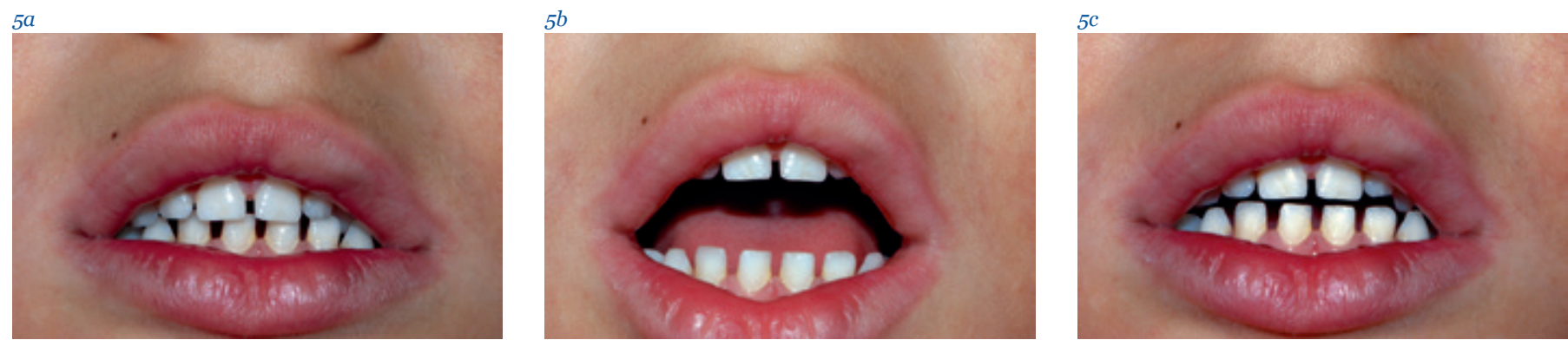

Fig. 5a-c Le linee mediane non sono coincidenti in massima intercuspidazione (a) ma lo diventano in apertura (b). In chiusura un precontatto deviante disloca lateralmente la mandibola (c) ripristinando la deviazione della linea mediana inferiore

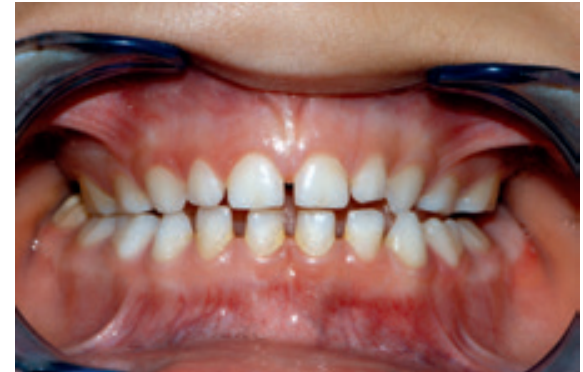

Fig. 6 Guidando la mandibola in relazione centrica le linee mediane superiori e inferiori si centrano reciprocamente e rispetto al centro del volto. In questa posizione è possibile individuare l'interferenza occlusale che causa la deviazione funzionale della mandibola. Questa si localizza generalmente a livello dei canini decidui

le interferenze occlusali che causano la deviazione funzionale (fig. 6).

\section{> Valutazione radiografica}

La valutazione delle proiezioni radiografiche nello studio delle deviazioni funzionali ha principalmente lo scopo di escludere una componente scheletrica nell'asimmetria in esame.

La teleradiografia del cranio in proiezione latero-laterale risulta di limitata utilità nello studio delle asimmetrie facciali, in quanto la sovrapposizione delle strutture della metà destra e sinistra del viso non permette una chiara lettura delle imma- gini. Tuttavia la presenza sulla pellicola di uno sdoppiamento dei margini inferiori del corpo mandibolare può essere dovuta a un dislocamento mandibolare funzionale [4,27-29].

La teleradiografia del cranio in proiezione postero-anteriore è considerato l'esame radiografico elettivo per la diagnosi delle asimmetrie facciali, in quanto le strutture del lato destro e sinistro del viso sono collocate alla stessa distanza rispetto alla pellicola e alla sorgente dei raggi X. L'analisi cefalometrica effettuata su tale proiezione permette di quantificare un'asimmetria di tipo strutturale. Attraverso alcune misurazioni trasversali e verticali, infatti, le strutture dento-facciali di un lato del viso vengono confrontate con quelle controlaterali [27,30-35]. L'ortopanoramica, infine, fornisce informazioni riguardo la forma della mandibola nelle sue componenti e permette di confrontare le due emimandibole in termini di lunghezza, altezza e livello occlusale dei denti $[17,21,22,26,27,36]$.

\section{$>$ Analisi dei modelli in gesso}

Attraverso l'analisi della simmetria condotta sui modelli in gesso è possibile valutare e confrontare lo sviluppo trasversale e sagittale delle due emiarcate.

L'analisi della simmetria trasversale è utile nei casi di cross-bite monolaterale, per distinguere una contrazione simmetrica dell'osso mascellare da una contrazione asimmetrica di tipo dentale o dento-alveolare. Entrambe le condizioni si manifestano clinicamente con una deviazione funzionale della mandibola in chiusura.

L'analisi della simmetria sagittale consente di individuare i casi di asimmetria dei rapporti sagittali di classe e di discriminare tra un'arcata sagittalmente asimmetrica - in cui è avvenuta una migrazione dentale monolaterale, conseguente per esempio alla perdita precoce di elementi decidui - e simmetrica, associata a una deviazione mandibolare funzionale in chiusura [37,38].

\section{CONCLUSIONI}

L'asimmetria mandibolare funzionale è una malocclusione che si manifesta nel soggetto in crescita, caratterizzata dalla laterodeviazione della mandibola in posizione di massima intercuspidazione, a seguito di precontatti devianti.

L'asimmetria funzionale è un quadro reversibile: infatti, se intercettata precocemente, si interrompono i meccanismi di adattamento che possono causare una crescita scheletrica asimmetrica.

L'analisi dei record diagnostici permette di individuare il fattore eziologico dell'a- 
simmetria funzionale e di pianificare una terapia che consenta di riequilibrare la crescita del paziente in una direzione armonica.

\section{CONFLITTO DI INTERESSI}

Gli autori dichiarano di non avere alcun conflitto di interessi.

\section{FINANZIAMENTI ALLO STUDIO}

Gli autori dichiarano di non aver ricevuto finanziamenti istituzionali per il presente studio.

\section{BIBLIOGRAFIA}

1. Ferrario VF, Sforza C, Poggio CE, Tartaglia G. Distance from symmetry: a three-dimensional evaluation of facial asymmetry. J Ora Maxillofac Surg 1994;52(11):1126-32.

2. Melnik AK. A cephalometric study of mandibular asymmetry in a longitudinally followed sample of growing children. Am J Orthod Dentofacial Orthop 1992;101(4): 155-66.

3. Hinds EC, Reid LC, Burch RJ. Classification and management of mandibular asymmetry. Am J Surg 1960;100:825-34.

4. Schmid W, Mongini F, Felisio A. A computer-based assessment of structural and displacement and asymmetries of the mandible. Am J Orthod Dentofacial Orthop 1991;100(1):19-34

5. Captier G, Lethuilier J, Oussaid M, Canovas $F$, Bonnel $F$. Neural symmetry and functional asymmetry of the mandible. Surg Radiol Anat 2006:28(4):379-86.

6. Kennedy DB, Osepchook M. Unilateral posterior crossbite with mandibular shift: a review. J Can Dent Assoc 2005;71(8):569-73.

7. Montaruli G, Caradonna C, Castellucci M, Ciccarelli E. Trattamento funzionale delle latero-deviazioni mandibolari. Mondo Ortodontico 2010;35(3):128-39.

8. Sato C, Muramoto T, Soma K. Functional lateral deviation of the mandible and its positional recovery on the rat condylar cartilage during the growth period. Angle Orthod 2006;76(4):591-7.

9. Fuentes MA, Opperman LA, Buschang $P$ Bellinger LL, Carlson DS, Hinton RJ. Lateral functional shift of the mandible: Part I. Effects on condylar cartilage thickness and proliferation. Am J Orthod Dentofacial Orthop 2003;123(2):153-9.
10. Nerder PH, Bakke M, Solow B. The functional shift of the mandible in unilateral posterior crossbite and the adaptation of the temporomandibular joints: a pilot study. Eur J Orthod 1999:21(2):155-66.

11. Ingervall B, Thilander B. Activity of temporal and masseter muscles in children with a lateral forced bite. Angle Orthod 1975:45(4):249-58

12. Ferrario VF, Sforza C, Serrao G. The influence of crossbite on the coordinated electromyographic activity of human masticatory muscles during mastication. J Oral Rehabil 1999;26(7):575-81

13. Kecik D, Kocadereli I, Saatci I. Evaluation of the treatment changes of functional posterior crossbite in the mixed dentition. Am J Orthod Dentofacial Orthop 2007;131(2): 202-15.

14. Alarcón JA, Martín C, Palma JC. Effect of unilateral posterior crossbite on the electromyographic activity of human masticatory muscles. Am J Orthod Dentofacial Orthop 2000;118(3):328-34.

15. Martín C, Palma JC, Alamán JM, Lopez-Quiñones JM, Alarcón JA. Longitudinal evaluation of SEMG of masticatory muscles and kinematics of mandible changes in children treated for unilateral cross-bite. J Electromyogr Kinesiol 2012;22(4):620-8.

16. Sonnesen L, Bakke M, Solow B. Bite force in pre-orthodontic children with unilateral crossbite. Eur J Orthod 2001;23(6):741-9.

17. Proffit WR. Ortodonzia moderna. II Ed. Milano: Masson, 2001.

18. Levrini A. Le abitudini viziate. Milano: Masson, 1989.

19. Cozza P, Mucedero M, Baccetti T, Franchi L. Treatment and posttreatment effects of quad-helix/crib therapy of dentoskeletal open bite. Angle Orthod 2007;77(4):640-5.

20. Mucedero M, Franchi L, Giuntini V, Vangelisti A, McNamara JA Jr, Cozza P. Stability of quad-helix/crib therapy in dentoskeletal open bite: a long-term controlled study. Am J Orthod Dentofacial Orthop 2013;143(5): 695-703.

21. Bracco P, Piancino MG. Terapia ortodontica in bambini affetti da crossbite monolaterale posteriore e open-bite: cicli inversi prima e dopo il trattamento. Ortognatodonzia Italiana 2007.

22. Piancino MG, Talpone F, Dalmasso P, Debernardi C, Lewin A, Bracco P. Reverse-sequencing chewing patterns before and after treatment of children with a unilateral posterior crossbite. Eur J Orthod 2006;28(5):480-4.
23. Brin I, Bren-Bassat Y, Blustein Y, Ehrlich J. Skeletal and functional effects of treatment for unilateral posterior crossbite. Am J Orthod Dentofac Orthop 1996;109:173-9.

24. Arnett GW, Bergman RT. Facial keys to orthodontic diagnosis and treatment planning-Part II. Am J Orthod Dentofacial Orthop 1993;103(5):395-411.

25. Bishara SE, Burkey PS, Kharouf JG. Dental and facial asymmetries: a review. Angle Orthod 1994:64(2):89-98.

26. Plint DA, Ellisdon PS. Facial asymmetries and mandibular displacements. $\mathrm{Br} J$ Orthod 1974;1(5):227-35.

27. Legan HL. Surgical correction of patients with asymmetries. Semin Orthod 1998:4(3):189-98.

28. Nanda R, Margolis MJ. Treatment strategies for midline discrepancies. Semin orthod 1996;2(2):84-9.

29. Cozza P, Ballanti F. Analisi cefalometrica diagnosi ortodontica. Roma: Società Editrice Universo, 2004

30. Peck S, Peck L, Kataja M. Skeletal asymmetry in esthetically pleasing faces. Angle Orthod 1991;61(1):43-8.

31. Letzer GM, Kronmann JH. A posteroanterior cephalometric evaluation of craniofacial asymmetry. Angle Orthod 1967;37(3): 205-11.

32. Cheney E. Dentofacial asymmetries and their clinical significance. Am J Orthodontics 1961:47:814-29.

33. Trpkova B, Prasad NG, Lam EW, Raboud D, Glover KE, Major PW. Assessment of facia asymmetries from posteroanterior cephalograms: validity of reference lines. Am J Orthod Dentofacial Orthop 2003;123(5):512-20.

34. Ricketts RM. Orthodontic diagnosis and planning. Angle Orthod 1982;5:351-70.

35. Ballanti F, Franchi L, Cozza P. Transverse dentoskeletal features of anterior open bite in the mixed dentition. Angle Orthod 2009;79(4):615-20

36. Fisher B. Asymmetries of the dentofacial complex. The influence on diagnosis, prognosis and treatment. Angle Orthod 1954;24:179-92

37. Cozza P, De Toffol L, Martucci L. Sviluppo della dentizione, guida all'occlusione, studio dei modelli in gesso. Roma: Società Editrice Universo, 2005

38. Portelli M, Matarese G, Militi A, Cordasco G, Lucchese A. A proportional correlation index for space analysis in mixed dentition derived from an Italian population sample. Eur J Paediatr Dent 2012;13(2):113-7. 